\title{
6 \\ Genes and Development: The Stem Cell Perspective
}

\subsection{Introduction}

The idea that genes control development is widespread in biology and philosophy. But the notorious ambiguity of the term "gene" makes assessment of this claim difficult. ${ }^{111}$ In classical genetics, "the gene" is a theoretical entity that predicts patterns of traits across generations. Localization of this hereditary material to chromosomal DNA and elucidation of its structure in the 1950s cemented the more realistic molecular gene concept. The Central Dogma (symbolized as DNA $\rightarrow$ RNA $\rightarrow$ protein) and genetic code then established molecular genes as the primary causes of phenotypes. Today, a common understanding of the term is:

(G) A gene is a causally active DNA sequence associated with a particular phenotypic trait.

This formulation leaves the nature of the association between genes and traits open. If it is causal, then we have:

$\left(G^{\prime}\right)$ A gene is a DNA sequence that causes a particular phenotypic trait. ${ }^{112}$

On this "causal-informational" view, a gene embodies the plan or program for a specific trait. It is then but a short step to the view that development is just the execution of a pre-determined genetic program. Twentieth-century triumphs in genetics have bolstered the view that genes are causal agents of distinctive significance for organismal development and resulting phenotypes. 
However, this "genetic exceptionalism" is vulnerable to two serious objections. The first is "Lillie's paradox:"113

It is... almost universally accepted genetic doctrine today that each cell receives the entire complex of genes. It would therefore appear to be self-contradictory to attempt to explain [differentiation] by behavior of the genes which are ex hyp. the same in every cell... The essential problem of development is precisely that differentiation in relation to space and time within the life-history of the individual which genetics appears implicitly to ignore... Those who desire to make genetics the basis of physiology of development will have to explain how an unchanging complex can direct the course of an ordered developmental stream.

(Lillie 1927, 365-367)

The problem can hardly be put more starkly. All cells of a multicellular organism (barring mutations and other rare exceptions) share the same DNA sequences. But development involves production of differences among an organism's cells, tissues, and organs. How can invariant genes account for developmental diversity?

The solution is to invoke differences in gene expression among cells and tissues. ${ }^{114}$ The Central Dogma represents gene expression as a simple causal-informational chain: a sequence of chromosomal DNA is a linear template for a mRNA transcript, which is, in turn, a template for a sequence of amino acids making up a protein. Cell phenotype depends on which genes are transcribed and then translated. But this raises a second objection: DNA does not express itself. Fifty years of molecular biology have revealed a menagerie of mechanisms implicated in the complex cellular machinery of gene expression. Transcription and translation are performed by elaborate protein-RNA complexes. The same chromosomal DNA may yield different proteins, depending on which other genes are expressed in a given cell. Chromosomal DNA also includes non-coding regulatory elements that affect gene expression: introns, promoters, termination sequences, upstream and downstream activators and enhancers, and multiple families of repetitive sequences. Non-DNA components of chromosomes, such as histones, modifier groups, and chromatin conformations, also influence gene expression. The path from DNA to protein is further complicated by mechanisms that modify gene products in multiple ways, including proofreading, RNA processing and editing, and alternative splicing. Yet another layer of RNA regulation was discovered in 2001: micro-RNAs, which modulate gene expression by specific 
sequence binding. Variation in gene products continues post-translation: proteins are spliced, folded, tagged for distribution within or without the cell, and cooperatively bind one another.

The complexity and diversity of gene expression mechanisms makes it impossible to identify any single molecular entity as a contextindependent gene $(\mathrm{G})$. What counts as a gene depends on the organismal and cellular context, as well as the trait of interest. Multiple layers of regulation allow for many ways of individuating "the gene," even for a particular protein. This inveterate pluralism vitiates the claim that genes $(\mathrm{G})$ control development. But a new defense of genetic explanatory privilege is available, building on the manipulability theory of causality (see Chapter 5). Its core thesis is that genes, but few other components of molecular mechanisms, are "specific, actual difference-making causes" of development (Waters 2007). I first unpack the terms of this thesis and then assess it in regard to stem cell biology.

\subsection{Genes as difference-makers}

According to the manipulability theory, causes are difference-makers. More precisely, $\mathrm{X}$ is a cause of $\mathrm{Y}$ just in case different values of a causal variable $\mathrm{X}$ make a difference to the value of an effect variable $\mathrm{Y}$, such that this relation is stable under some interventions. So only the terms "specific" and "actual" require clarification here. Waters (2007) defines the latter as follows: $\mathrm{X}$ is the actual difference-maker with respect to trait $\mathrm{Y}$ in population $\mathrm{P}$ if and only if $\mathrm{X}$ causes $\mathrm{Y}$ in Woodward's sense; this relation is invariant with respect to other variables that actually vary in $\mathrm{P}$; the value of $\mathrm{Y}$ actually varies among members of $\mathrm{P}$; and actual variation in $\mathrm{X}$ fully accounts for the variation in $\mathrm{Y}$ in P. ${ }^{115}$ These conditions, he argues, mark an "objective difference" between actual and merely potential difference-makers. Waters' account acknowledges the molecular complexity of gene expression in that "DNA is only one of many causes" of biological phenomena and "exercises its roles through the production of RNA and polypeptide molecules" (2007, 552-553). However, not all causes of development are ontologically "on par." Genes, as actual difference-makers, are responsible for phenotypic variations among members of actual populations.

This objective difference among biological causes underwrites an explanatory privilege for genes in development. The effect explained is a difference in the value of a variable $\mathrm{Y}$ in some population of developing entities. The explanatory cause is a variable $\mathrm{X}$ that can take multiple values and satisfies the conditions for an actual difference-making cause. 
Differences in DNA sequence that correlate with different phenotypic traits in a real population meet these criteria. In contrast, molecular and cellular entities that make a causal contribution to development, but do not vary within the population of interest, are merely potential difference-makers. Genes $(G)$ are therefore distinctively responsible for actual differences in a population of cells or organisms. This conception of genes dates back to the early twentieth century and T. H. Morgan's research program of classical genetics. Morgan conceived the difference in many-to-many relations between genes and traits as the attribute solely of the gene $(1926,322)$. His differential gene concept accords with the manipulability theory. Both shift the focus of explanation from the nature of a cause to what makes a difference to the value of a variable under controlled conditions. Experiments designed to reveal causal relations pick out difference-making causes. ${ }^{116}$ Waters' application of the manipulability theory is thus supported by classical genetics.

However, being constrained to accommodate molecular genetics as well, Waters' account limits genetic explanatory privilege in several ways. First, genes are actual difference-makers only relative to a population. This restricts gene-based explanations to a particular genetic and environmental context, within which "uniform phenotypic differences" are caused by differences in DNA (Waters 2007, 553). Second, the explanandum for which genes offer a privileged explanation is a difference among members of a population, not development per se. Finally, Waters' argument concerns only the "small part of development" described by the Central Dogma, namely specification of RNA/protein sequence by DNA sequence. So his defense of genetic explanatory privilege is restricted to the "coding" relation between a population of DNA sequences and a population of RNA or protein molecules within a cell.

This difference-making relation has another distinctive feature: specificity. DNA is distinctive, Waters argues, in that different specific changes to its sequence produce different specific changes in molecular products (2007, 574-575). Other components of cellular machinery relate to the latter more simply, like on/off switches. DNA's specificity permits finer modulation of phenotypic states. Woodward (2010) further explicates this concept. ${ }^{117} \mathrm{~A}$ classic illustration is the lock and key model of enzyme action (see §5.6). In this (disconfirmed) model, each enzyme binds a unique substrate to catalyze a unique chemical reaction. A single cause (enzyme) produces one kind of effect (reaction), while each effect is produced by a single cause. Specificity in this "one cause-one effect" sense is relative to a pre-specified class of alternative causes and a pre-specified range of kinds of effect. Because there are no fixed criteria 
for distinguishing among alternative causes and bounding the ranges of effects, this concept remains somewhat vague.

Further clarity is provided by a second specificity concept, defined in terms of a mapping from states of a cause $\mathrm{X}$ to states of an effect $\mathrm{Y}$ (Woodward 2010, 305). The more closely this mapping approximates a bijective function, the more specific the causal relation between $\mathrm{X}$ and Y. The key idea here is "proportional influence" (hereafter PI) of causes on effects. If we can vary the state of the cause, then we can determine which state of the effect is realized, out of a range of alternatives. Nonspecific causes are such that many different states of $X$ map to the same state of $Y$, or the same state of $X$ maps to many states of $Y$, or both. So, like 'actuality,' PI-specificity provides a basis for discriminating among the various causes of an effect. Relative to sets of alternative values of an effect variable and each of its causal variables, more PI-specific causes afford finer-grained control over the value of the effect. If finegrained control is advantageous for explanation then there is reason to privilege PI-specific causes in explanations over non-PI-specific causes of the same effect. These two concepts of causal specificity, PI- and one-cause-one-effect, are not precisely equivalent. ${ }^{118}$ But, for present purposes, they can be treated together. The explanations at issue are mechanistic explanations (MEx). In constructing these explanations, the overall mechanism, associated phenomena, and components are delineated, thereby fixing the sets of alternative values to be considered. Relative to such a set, one-cause-one-effect and PI-specificity coincide. For simplicity, "PI-specificity" hereafter refers to both concepts.

Augmented with the concept of PI-specificity, Waters' view amounts to a sophisticated form of genetic reductionism. The key idea is that, though MEx describe many causal relations, those involving genes and their effects (RNA and protein sequences) are distinguished by PI-specificity. In this sense, genes are fundamental to MEx of development. Unlike linguistic and information-theoretic treatments, PI-specificity casts DNA as a fundamental template on the basis of causal relations relevant to experiment. This is a promising idea. The next two sections critically examine this sophisticated gene-centric view.

\subsection{Specificity and mechanistic explanation}

PI-specificity bears on MEx in several ways. First, PI-specific causal relations are good starting-points for these explanations. This is because PI-specific input-output relations indicate the boundaries of a mechanism of interest, while conditions that reveal PI-specificity often indicate 
key components. Second, PI-specificity guides construction of MEx, identifying relevant components and the appropriate grain of description for their workings. Third, PI-specificity motivates MEx in practical contexts. Biotechnology, for example, often exploits PI-specific causal relations in mechanisms that we construct. But to intelligently use a PI-specific causal relation we need to know how its mapping is achieved. If we know how a fine-grained mapping of input and output conditions is brought about in one context, we can make inferences about its stability in other contexts, as well as possible unintended consequences. Furthermore, as mechanisms are often components of other mechanisms, causal relations within mechanisms we engineer are often PI-specific.

These points notwithstanding, PI-specific causal relations are neither necessary nor sufficient for MEx. That they are insufficient is straightforward. The enzyme RNA polymerase I (hereafter RNA pol I) is a crucial component of the mechanism by which a DNA sequence is transcribed to produce a mRNA. Many different states of this protein are possible, which vary in sequence and shape. But states of RNA pol I do not map onto states of transcribed mRNA so as to approximate a bijective function. Many different states of RNA pol I are compatible with its functional role and, from each of these, many mRNA sequences can be produced. Many other states are non-functional and all map to the same effect: no mRNA. So RNA pol I is non-PI-specific. Yet no adequate MEx of transcription could omit this protein. Description of actual PI-specific difference-making causes is not sufficient.

Nor is PI-specificity necessary for MEx. Consider the lock-and-key model again. In mechanistic terms, the lock and key relation holds not between enzyme and chemical reaction, as in PI-specificity, but between enzyme and substrate. Binding of these two components into a complex catalyzes one or more chemical reactions. Many experiments have shown that the causal relation between enzymes and reactions is not PI-specific. But enzymatic reactions are well understood mechanistically. Enzymes and substrates bind specifically to one another in virtue of certain molecular features. As discussed in Chapter 5, binding 'partners' have complementary shapes and biochemical properties that, given certain spatio-temporal conditions, cause them to form a complex via weak chemical bonds. Enzyme-substrate complexes play a distinct causal role, which the same components, dissociated, cannot. Lack of PI-specificity does not undermine MEx of enzyme catalysis.

It is even possible that PI-specific causal relations are the exception rather than the rule in biological mechanisms. Redundancy, multiple 
interactions per component, and mutual adjustment of parts are all characteristic of developmental mechanisms, and all involve deviations from PI-specificity. MEx of developmental phenomena remain incomplete, despite enormous progress in identifying genes and PI-specific molecular relations, precisely because of the prevalence of non-PI-specific relations in development. A different concept of specificity, concerning organization of interactions among heterogeneous components, may be more relevant for MEx. As the previous chapter showed, the overall working of a mechanism typically depends on exactly which components interact with one another, as well as when, where, and in what context. Mechanistic descriptions must be specific in this respect. But this is distinct from PI-specificity. Hereafter, the term "specificity" refers to the interactive, organizational conception.

Though description of PI-specific causes is neither necessary nor sufficient for MEx, such explanations do describe actual difference-makers. MEx aim to describe how some mechanism actually works, or, sometimes, how a phenomenon $\mathrm{P}$ is actually brought about. Experiments that reveal the answers show that manipulating a component makes a difference to the overall mechanism and to interactive processes within it. So both an overall mechanism and its components are actual difference-makers. But the crux of MEx is not identifying difference-makers as such. Rather, a MEx shows how interacting components jointly constitute the overall mechanism, unifying levels of biological organization in a particular context (see §5.6). MEx do not show that some mapping from cause to effect exists, but describe how the mapping is made. Within a MEx there is explanatory parity; all components are equally implicated. So the actual/potential and PI-specific/non-PI-specific distinctions do not underwrite a privileged role for genes $(G)$ in MEx of development, though they may do so in other kinds of explanation.

\subsection{Cell reprogramming}

I next consider a second argument for genetic privilege, based on cell reprogramming experiments. Reprogramming experiments manipulate cell development by adding specific DNA sequences to cultured cells. The first reprogramming experiments used four genes (Oct3/4, Sox2, Klf4, and c-Myc) and mouse skin cells (see §2.5.1). The genes were "delivered" to cells using engineered retroviruses, which infected cells and integrated new DNA sequences into their chromosomes. A very small percentage $(\sim 0.05 \%)$ of cultured cells then transformed their morphology, gene expression, and developmental capacities, becoming similar to 
embryonic stem cells (ESC). These 'reprogrammed' cells can self-renew indefinitely or give rise to many different cell types. They are accordingly termed 'induced pluripotent stem cells' (iPSC). Reprogramming evidently makes a difference among cells, and it is reasonable to suppose that DNA sequences are the actual difference-makers in this case. But, surprisingly, they are not. The actual difference-makers in reprogramming experiments are complex molecular mechanisms that include, but are not controlled by, particular DNA sequences.

The first hint that more is involved than difference-making DNA is that reprogramming with genes is "a slow and inefficient process consisting of largely unknown events" (Maherali and Hochedlinger 2008, 595). At best, only a small percentage $(<0.1 \%)$ of gene-treated cells become iPSC. Difference-making relations do hold at the population level. But the striking inefficiency of reprogramming for individual cells highlights our lack of understanding of how the process works; that is, the mechanism by which cells are transformed. DNA components of the mechanism, the four reprogramming factors, are well characterized and can be precisely controlled. But these genes are not sufficient. Experiments aimed at revealing reprogramming mechanisms therefore target other components and interactions as well: levels and timing of gene expression within cells, biochemical interactions that affect chromosome structure, intercellular signaling pathways, and more. Since 2006, the original iPSC method has been refined and modified by hundreds of different laboratories. The result is not a single standardized procedure, but a multistage schema indicating variables that make a difference to experimental outcome (Figure 6.1). ${ }^{119}$ Five kinds of variable are distinguished: (i) reprogramming factors, (ii) delivery method, (iii) starting cell population, (iv) factor expression, and (v) culture conditions. Different combinations of values of these five variables are more or less effective at producing iPSC with desirable developmental capacities, such as the ability to produce neurons, blood, or cardiac muscle in vitro. ${ }^{120}$

For any particular experiment, values of these variables are selected according to researchers' purposes and mutually optimized, insofar as current knowledge permits. If reprogramming is conceived as a causal process that makes a difference to cells in the starting population (iii), then variables (i), (ii), (iv), and (v) are all actual difference-makers. Moreover, DNA is not required for (i). RNA and protein sequences can also reprogram cells to iPSC. So progress in reprogramming since 2006 does not single out DNA sequences as the actual difference-makers. Instead, the emerging picture is of diverse molecular mechanisms involving multiple interacting variables. 


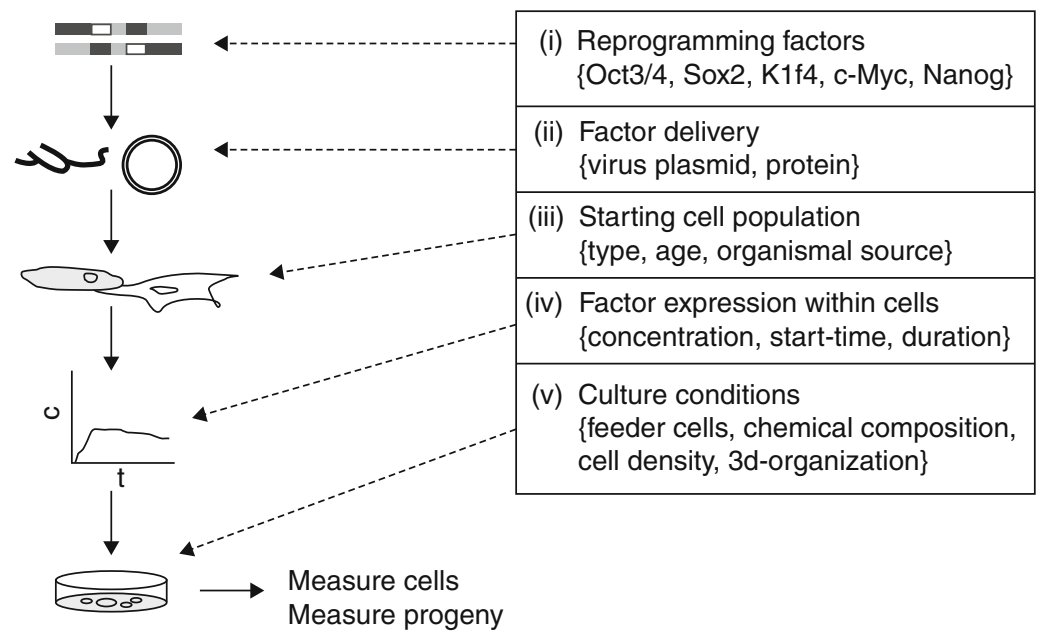

Figure 6.1 General scheme of iPSC reprogramming

Furthermore, genes were not actual difference-makers in the original 2006 experiments that first yielded iPSC. Researchers at Kyoto University began with the hypothesis that "the factors that play important roles in the maintenance of ES cell identity also play pivotal roles in the induction of pluripotency of somatic cells" (Takahashi and Yamanaka $2006,663)$. They used this hypothesis to construct a list of 24 candidate factors, including genes and proteins. Genes highly expressed in tumors and ESC were selected, as were proteins implicated in pluripotency mechanisms in embryos and ESC. ${ }^{121}$ Genes, as such, were not given priority as candidates to induce pluripotency. Instead, all three molecular forms in the Central Dogma - DNA, RNA, and protein - were subsumed under the inclusive term "factor." Next, the 24 candidates were whittled down to a "core set:" Oct3/4, Sox2, Klf4, and c-Myc. Each member of the core set was characterized both as DNA and as protein (Yamanaka 2007, 43-45). So throughout the experiment proteins were as significant as DNA. Difference-making factors were individuated and tested in ways that did not discriminate among these molecular forms.

In addition, the method by which the core set was generated is designed to reveal jointness rather than PI-specificity. First, the Kyoto team showed that all 24 candidates together are sufficient to induce pluripotency. Next, they added each factor to cells individually, which yielded no iPSC. This demonstrated that interactions among factors are necessary to make a difference to cell development; "drastic alterations 
of cell fate could be achieved with a combination of factors when no single factor would suffice" (Cohen and Melton 2011, 248). Subsequent experiments were designed to identify the key participants in these interactions. Each candidate was subtracted individually for a total of 24 different combinations of 23 factors. Ten of these yielded no iPSC, indicating that the missing factor in each case is necessary to induce pluripotency. When these 10 essential factors were combined, they induced pluripotency more efficiently than the entire set of 24. "Individual subtraction" experiments were then performed for each essential factor, revealing four as the essential core set. These experiments demonstrated not only that interactions among multiple factors are necessary to make a difference to cell development, but also that the key interactions are contextdependent. The essential factors in the set of 24 are not the same as the essential factors in the set of 10 , and the latter combination is more effective than the former. Overall, the design and results of iPSC experiments conform well to the joint account of MEx.

Although current MEx of reprogramming are works-in-progress, some robust features have already emerged. Most strikingly, all members of the original core set, and all comparably effective alternatives, are transcription factors (TF). As proteins, TF bind specifically (in the mechanistic sense) to particular sequences of DNA, which, in turn, affects transcription of genes nearby on the chromosome (Figure 6.2). TF-binding DNA sequences are regulatory rather than protein-encoding; the mechanism of transcription involves both. These regulatory sequences are distributed non-randomly in the genome; notably, the same sequence often appears near genes with related functions. So altering the expression of one TF gene can make a difference to the expression of dozens, even hundreds, of other genes - including other TF genes. TF proteins are thus "phenotypic switches" that can coordinate large-scale patterns of gene expression within a cell. ${ }^{122}$ The crux of coordinated switching is the binding interaction of a TF protein and a regulatory DNA sequence. Neither component has priority over the other; both DNA and protein are crucial. In emerging MEx of cell reprogramming, DNA sequences play a significant role - but not a controlling one. Genes are interactive partners with other components, rather than "masters" dominating minions.

Reprogrammers' terminology is also inconsistent with the idea that genes are privileged components of developmental mechanisms. In experimental biology, by convention, names of genes are written in italics, names of proteins in regular type (for example, Nanog and Nanog). ${ }^{123}$ Though stem cell biologists typically respect this convention, discussions of reprogramming often shift rapidly between DNA and protein terms, 


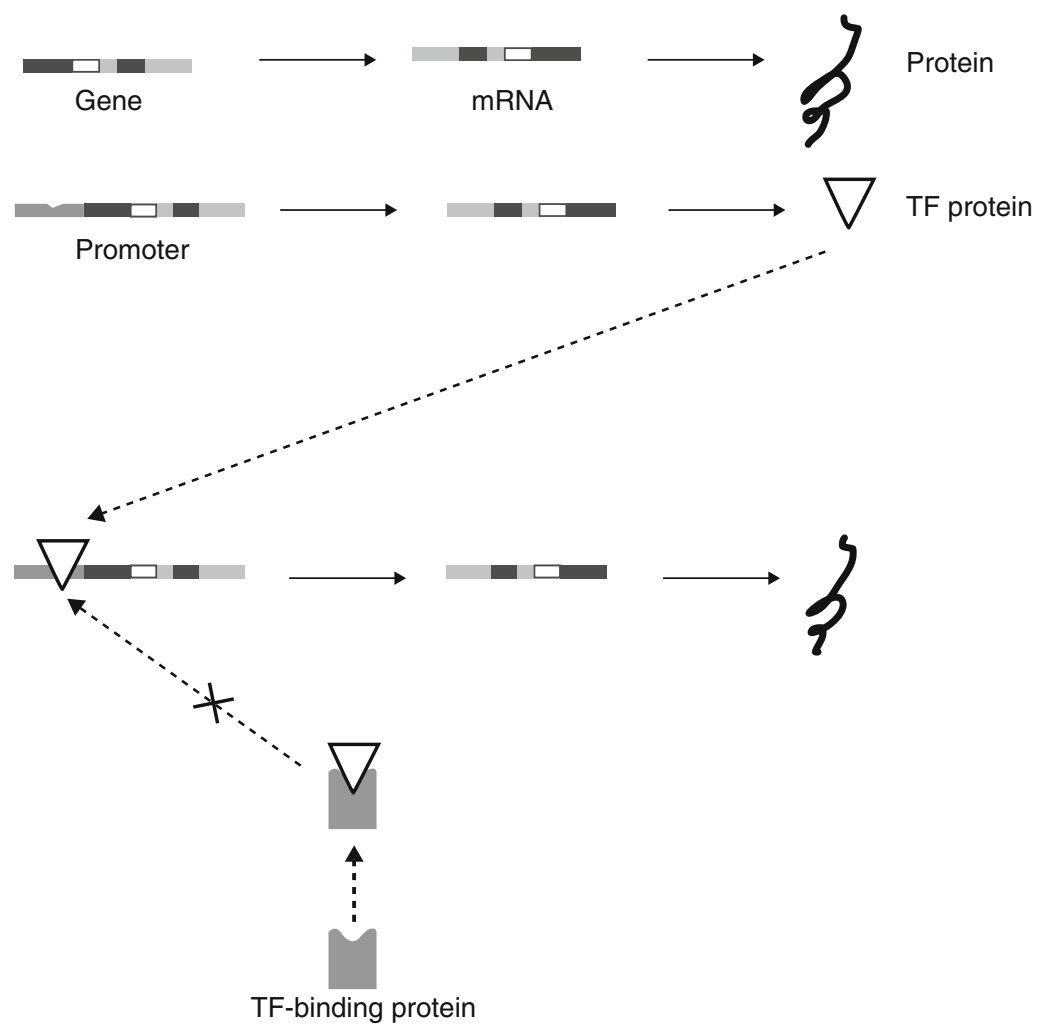

Figure 6.2 Role of transcription factors

or use regular type only. ${ }^{124}$ Terms such as 'Oct3/4' may refer to that TF's DNA sequence, RNA transcript, or functional protein. Indeed, the inclusive term 'factor' is plausibly taken as referring to all three, given that the mechanistic role of TF is to mediate between DNA, RNA, and protein. On this interpretation, 'Nanog,' for example, refers to an entire TF unit: a compressed version of the Central Dogma.

Increased understanding of TF mechanisms has led to a shift in the concept of reprogramming itself. Intuitively, the term suggests a preexisting plan for cell development, which experimenters reprogram by adding exogenous TF. But informational metaphors are neither prevalent nor powerful in stem cell biology today (Brandt 2010). "Cell reprogramming" refers generally to experimental methods that induce pluripotency. There are four such methods; iPSC production by TF being the newest. Two of the methods, nuclear transplantation and cell fusion, 
were pioneered in the 1950s. In the former, a mature cell's nucleus is transplanted into an undifferentiated egg; in the latter, a differentiated cell is fused with an undifferentiated cell. Both can induce early development in a cell with an 'old' nucleus. ${ }^{125}$ Reprogramming was thus originally conceived as an effect of cytoplasm on the nucleus; one part of the 'intracellular geography' affecting another. The other methods, invented more recently, induce pluripotency in cultured cells by either growing cells atop one another or adding specific TF. Such experiments show effects of external factors, whether from other cells or an experimenter's syringe, on an entire cell. So it is cells, rather than nuclei, that are reprogrammed. ${ }^{126}$

The two senses of reprogramming involve different conceptions of gene expression. The earlier concept suggests a privileged role for genes in development. On this conception, the nucleus is reprogrammed by cytoplasmic factors, resulting in changed gene expression and, through gene action, altered development. Cytoplasmic factors affect development only by way of the nucleus, where gene action is localized. Effects then emanate 'outward' along the linear path of the Central Dogma. The second concept associates gene expression with cell state, which encompasses molecular, cellular, and developmental traits (Chapter 4). Molecular traits are thought to underlie, and thus mechanistically explain, the others (Chapter 5). The underlying 'circuitry' that maintains a cell state consists of DNA, RNA, protein, and small molecules, collectively referred to as a 'program.' Reprogramming, in this sense, refers to the process of changing a cell's state by manipulating the underlying molecular program.

TF play a distinctive role in these programs, acting as 'switches' for manipulating cell state. TF can therefore qualify as actual differencemakers for cell development. But they are not PI-specific. The relation between TF and cell state is many-to-many. Moreover, TFs do not act alone, but jointly. Explanations of differential developmental outcomes go beyond identification of TF, to describe entire mechanisms. For example, three TF (Oct4, Sox2, and Nanog) are proposed as a "core pluripotency network" that can act as a switch for developmental potential in mouse and human cells. This network is thought to play two key roles: repress genes associated with differentiation, and activate ESC-specific genes (Stadtfeld and Hochedlinger 2010, 2249). Preliminary MEx of both activation and repression describe binding of protein complexes to "cognate DNA sequences." DNA sequences are not privileged in these explanations. Instead, emphasis is on specific interactions among components, that is jointness. 
To sum up: the concepts of reprogramming, cell state, and mechanistic explanation are closely connected in stem cell biology today. Reprogramming is increasingly conceived as change in cell state, though the earlier sense of the term also persists. Cell state implicates both molecular and cellular levels, giving the new sense of reprogramming a dual character. The two levels correspond to the multilevel structure of MEx for cell development, as explicated by the joint account (Chapter 5). Mechanisms of cell reprogramming are constituted by diverse components that selectively associate so as to jointly bring about a change in cell state. Within these mechanisms, all components are equally crucial; none is privileged over others. Genes do not play a controlling or foundational role in MEx of cell state changes. The reprogramming case does not support the view that genes play a privileged role in development as actual, specific difference-makers.

\subsection{Waddington's epigenetic ${ }^{127}$ landscape}

A better representation of genes in development, with particular relevance for stem cell phenomena, is Waddington's landscape. Conrad Hal Waddington articulated the landscape metaphor for development in several texts, most extensively in The Strategy of the Genes (1957). ${ }^{128}$ This simple model, originally constructed to unify embryology and genetics, has been recently co-opted by stem cell biologists to represent reprogramming experiments. The landscape's structure, representational assumptions, and relation to experiment, in both original and updated versions, clarify the role of genes in development.

The landscape model is a two-dimensional diagram of a threedimensional structure (Figure 6.3):

...a more or less flat, or rather undulating surface, which is tilted so that points representing later states are lower than those representing earlier ones... Then if something, such as a ball, were placed on the surface, it would run down toward some final end state at the bottom edge.

(Waddington 1957, 29)

The axis projecting outward to the viewer represents time. The horizontal axis represents phenotype, ordered by some measure of similarity. The vertical axis represents the order of development; the surface's tilt correlates this developmental order with time. A rolling ball's path down the incline corresponds to development of some part of an organism 


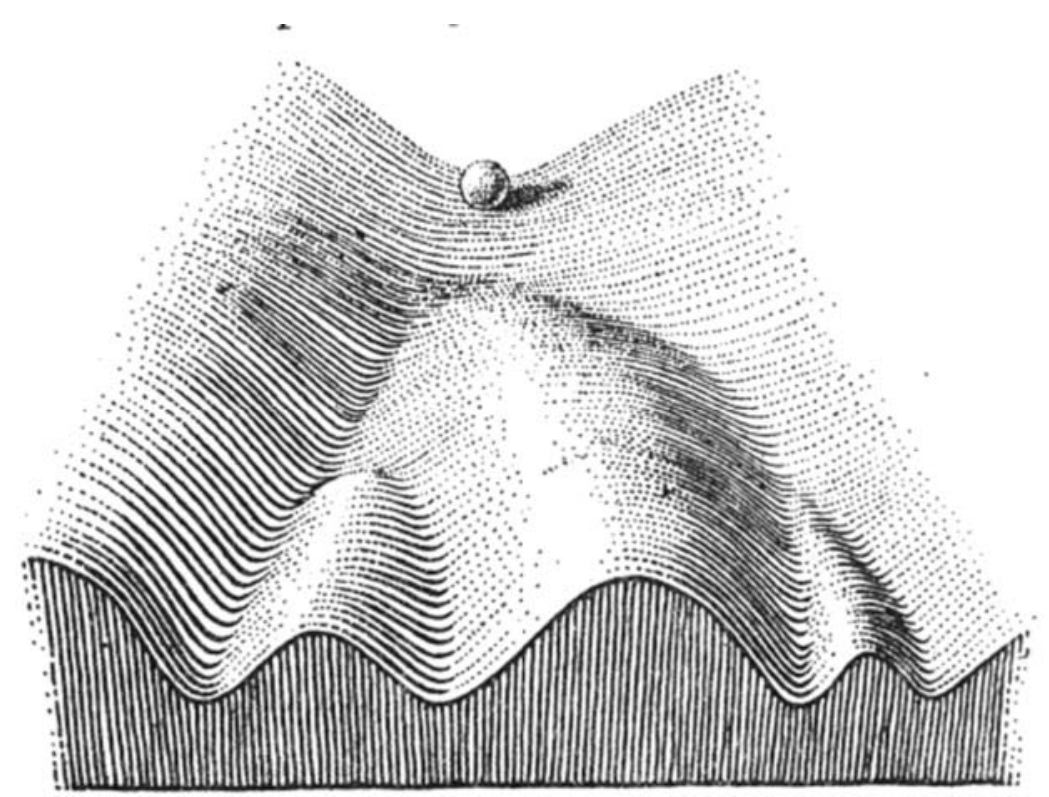

Figure 6.3 Waddington's landscape model (from Waddington 1957, 29)

from an early undifferentiated state to a mature differentiated state. The bottom edge describes a series of dips representing alternative mature states, while the top edge describes a curve with a single minimum, representing the undifferentiated start of development. The undulations of the landscape carve it into valleys with the form of branching tracks. These valleys connect the initial state with multiple discrete end-states. So the landscape represents developmental potential gradually restricted over time, partitioned into diverging channels. One could say that it diagrams the concept of 'normal development' as a simple geometric structure. For Waddington, the model served three related purposes, which are reflected in its structure and representational assumptions. I discuss each in turn.

\subsubsection{Developmental pathways}

Most obviously, the landscape model represents patterns of phenotypic change in developing tissue. Prominent valleys represent resistance of these patterns to external perturbation: robustness. The developing entity itself is left unspecified; ${ }^{129}$ the pathways of development are the main representational target. In other words, Waddington's landscape represents 
developmental potential rather than development per se. The ball is poised at the top, with the landscape fanning out below. In this position, the ball represents a fertilized egg or a portion thereof; Waddington does not specify an interpretation for other positions. Indeed, the distinction between surface and ball is somewhat arbitrary. In a 1956 diagram, Waddington identifies the top edge as representing an early developmental stage, with different points representing "intrinsically different" regions of egg cytoplasm $(1956,351)$. Alternatively, and equivalently, different regions of egg cytoplasm could be represented as balls having different initial biases that influence their trajectories. This flexibility makes it difficult to specify the representational target of the ball at intermediate positions, other than generically, as 'the developing entity.' But this interpretative difficulty simply reinforces that the developing entity is not the model's representational target. Rather, Waddington's landscape represents developmental pathways, the pre-determined options available to the fertilized egg or a part thereof.

This representational target can be analyzed further. The landscape model exhibits three "essentially formal" properties of development: unidirectionality in time, multiple discrete termini from a single undifferentiated start, and robust bifurcating tracks $(1957,49)$. These structural features of the model reflect generalizations about animal development, which are, in turn, based on empirical observations and experiments. Unidirectionality in time and multiple discrete termini from an undifferentiated start-point are features taken as essential to organismal development, supported by observations dating back to Aristotle. Robustness and bifurcating structure of developmental pathways, however, are generalizations grounded in twentieth-century experimental embryology. The model represents two such generalizations: first, that developmental pathways tend to "self-stabilize" in the face of minor perturbations and, second, that certain steps in developmental pathways depend on a (nonspecific) stimulus. Waddington inferred both from the combined results of experiments on Drosophila, chick, and various marine organisms. The first generalization is based on experiments that varied the physical or chemical environment of a developing embryo. Results showed that for many such interventions, not only developmental outcomes, but also intermediate stages, remained constant. ${ }^{130}$ Waddington concluded that developmental pathways tend to self-stabilize in the face of minor perturbations of internal or external origin. Their robustness is represented in the model by valleys' depth and steepness. A valley with steep walls corresponds to an interval of a developmental process in which the fate of the tissue in question is pre-determined, barring extreme perturbations. 
A valley with gently sloping walls corresponds to an interval in which the tissue is responsive to internal or external stimuli, such that small disturbances can 'push' development onto another path.

Other embryological experiments indicated that developmental pathways include intervals of stimulus-dependence. For example, in chick and Drosophila, neural ectoderm forms an eye lens only if an inducer is present at a particular time; otherwise, no lens develops. During the crucial interval, diverse substances - other tissues, chemicals, objects introduced by the experimenter, even artificial compounds that would never be encountered in normal development - can induce lens formation. So the inducing substance does not specify the fate of the developing tissue. These results were obtained by experiments that isolated parts of a developing organism and then added or removed certain environmental factors at particular times. To explain the results, Waddington distinguished between a tissue's competence to respond to an inducing stimulus and its potency, or ability to give rise to a range of specific developmental outcomes. The developmental potency of neural ectoderm, at least in some organisms, is determined in advance as a choice of two options. Which of the two is chosen depends on the presence or absence of a stimulus when the tissue is in a competent state. Alternative pathways are represented in the model as bifurcating tracks. Waddington used the landscape analogy to illustrate the distinction between competence and potency for developmental processes in general. ${ }^{131}$ So the model's prominent structural features, notably its branching valleys, are grounded on experiments that manipulated development in model organisms.

\subsubsection{Genetic control}

Waddington's landscape also expresses a hypothesis about the role of genes in development: that genes indirectly control development through a network of interacting biochemical products. The idea is depicted in a companion diagram showing the 'underside' of the landscape, which reverses the viewer's perspective (Figure 6.4). Seen from below, the landscape's surface "slopes down from above one's head towards the distance" $(1957,36)$. The contours of the landscape are "controlled by the pull of these numerous guy-ropes which are ultimately controlled by genes." Guy-ropes represent gene products, while their webby connections represent biochemical interactions among those gene products. The underside diagram shows gene products organized into interacting networks that directly determine the landscape's topography. The ultimate determinants, however, are genes, depicted as fixed pegs. Their fixity represents genes' lack of alteration during development, and suggests a 


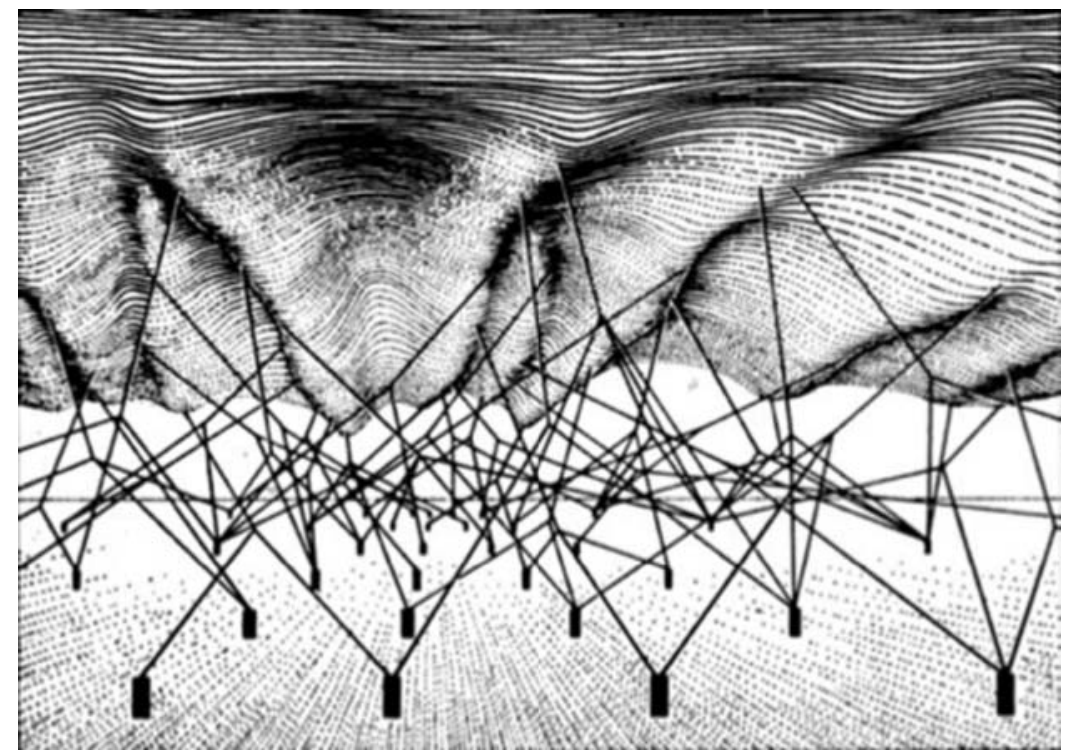

Figure 6.4 Genetic underpinnings of Waddington's landscape (from Waddington $1957,36)$

kind of ultimate control. Waddington's hypothesis about genetic control of development is visualized by the landscape's two sides: a top-side view of branching pathways leading to stable developmental states, and an underside of genes and their interacting products.

The model visually unifies development and genetics via these two complementary images of the landscape: robust pathways above and interacting gene products below, with genes at the bottom metaphorically 'pulling the strings.' Development and genetics are further unified via the branching-track structure, which visualizes a formal analogy between cellular, genetic, and organismal development. Branching tracks have been used to represent cell development since the late nineteenth century, most prominently in cell lineage diagrams that track cell pedigrees and division events. ${ }^{132}$ In these models, branch-points represent cell division events and branching tracks represent genealogical relations among cells. Other cell characteristics are also represented, such as position within the developing embryo, morphology, and developmental fate. Waddington's landscape is evidently not a cell lineage diagram, as it does not represent cell properties, intercellular relations, or, indeed, cells at all, apart from the fertilized egg (see note 129). The landscape's branch-points 
represent choices among developmental pathways, not mitotic events. Nonetheless, as Gilbert (1991) persuasively argues, the structural correspondence between cell lineage diagrams and Waddington's landscape draws a formal analogy between cell and organismal development.

The structural correspondence with models of gene action goes deeper. Waddington first articulated the landscape analogy in 1939 as a generalization of time- and dose-effect curves representing the role of genes in producing specific phenotypic effects. In these genetic models the effects of different alleles of a single gene are plotted against time, with "doses" differing for different combinations of alleles. Waddington modified these diagrams to include branch-points, representing steps in a biochemical pathway at which differences in a gene's product make a difference to the phenotypic result $(1939,182)$. For example, Drosophila eye color results from a pathway such that the presence or absence of a particular gene product makes a difference to the pigmentation in the adult fly. Generalizing from data on Drosophila mutants, Waddington hypothesized that genetically-controlled switch-points underlie the developmental pathways that produce phenotypic traits. The bifurcations of developmental pathways and genetically-controlled switch-points are thus different aspects of the same process.

'Branching' representations of gene action depict the difference that mutant versus wild-type alleles of a gene make to phenotypes, while branching tracks on the landscape represent the possibilities available to developing tissues. Because multiple genes were usually implicated in a single pathway, such as that producing Drosophila eye color, branchingtrack models naturally expanded to include effects of multiple interacting genes. The landscape model results from including interactive effects of the entire genome on a particular pathway:

If we want to consider the whole set of reactions concerned in a developmental process such as pigment formation, we therefore have to replace the single time-effect curve by a branching system of lines which symbolizes all the possible ways of development controlled by different genes. Moreover, we have to remember that each branch curve is affected not only by the gene whose branch it is but the whole genotype. We can include this point if we symbolize the developmental reactions not by branching lines on a plane but by branching valleys on a surface. The line followed by the process, i.e. the actual time-effect curve, is now the bottom of a valley, and we can think of the sides of the valley as symbolizing all the other genes which cooperate to fix the course of the time-effect curve; some of these genes will belong 
to one side of the valley, tending to push the curve in one direction, while others will belong to the other side and will have an antagonistic effect. One might roughly say that all these genes correspond to the geological structure which moulds the form of the valley.

(Waddington 1939, 182, emphasis mine)

\subsubsection{Unification}

Waddington's branching-track representation of gene action was speculative, conforming to his unificatory aims rather than contemporary standards in genetics (Gilbert 1991). These unificatory aims also extended to evolution. To bring evolution into the picture, Waddington conceived the landscape as malleable, such that changes to a gene or interactions among products alter its topography. These changes represent modification of developmental pathways on evolutionary time-scales. Waddington further speculated that developmental pathways bias genetic change in particular directions, forming a feedback loop of evolutionary and developmental change mediated by genes. He intended the landscape as a "conceptual laboratory" for visualizing theoretical ideas about these interrelated processes of change. One influential example is canalization, the process by which developmental pathways become more robust to perturbations. The more canalized a pathway, the more stimuli can induce a tissue to enter it and the less stimuli can turn a tissue from it. Canalization is visualized on the landscape as increased steepness of valley walls. In the model, such topographic changes are produced by changes in the underlying network of interacting genes and products: changes in the placement of pegs or tensions on the guy-ropes. So the model offers an intuitive illustration of evolution of development via genetic change. Malleability of the landscape is crucial to such illustrations, representing interrelated developmental, genetic, and evolutionary processes.

Its role as conceptual laboratory also constrained the landscape model to be simple. Waddington was well aware that a rigorous treatment of development in a geometrical framework would add a dimension for each phenotypic trait, with each point representing a complete state description of the developing entity in multi-dimensional space (1957, $26,49)$. Yet he constructed the model in three dimensions. Phenotypes are represented on a two-dimensional surface, one dimension of which is correlated with time. So features of the model that could be used to derive predictions (such as steepness of valley floor or walls compared with the overall slope of the landscape) lack a principled theoretical interpretation. The rate of developmental change over time or the degree 
of a pathway's robustness, for example, can be represented only qualitatively, on an arbitrary scale. As a consequence, Waddington's landscape cannot be used to derive specific predictions about developmental mechanisms or their genetic control. Despite its geometric structure, the landscape model is non-mathematical, inexact, and qualitative. Its role is not to predict or explain, but to speculate and explicate. The restriction to three dimensions allows intuitions shaped by everyday experience to be brought to bear on aspects of development, genetics, and evolution as represented in the model. The price of this intuitive picture of canalization and other evolutionary developmental processes is rigor and precision. As few molecular details were available at the time, this was an easy trade-off for Waddington. The landscape model was intended for "a context in which it is more important to employ a system of thought which is flexible and of wide application than to search for a precise formulation of a narrower viewpoint" $(1957,31)$. To play its unificatory role, the model was constrained to be simple, rather than an accurate or principled representation of animal development.

To sum up: Waddington's landscape offers a convenient diagrammatic framework for conceptualizing the role of genes in development. It is simple, bears a clear relation to experiments, and represents genetics and development as complementary approaches, unified by structural correspondence. It also represents genes as controlling development: the fixed ground of developmental potential. As argued above, this last feature is no longer applicable. Modifications to the landscape introduced by cell reprogrammers bear out this claim.

\subsection{Reprogramming and the landscape}

Unusually for a product of the 1950s, Waddington's landscape appears in several high-profile reviews and commentaries on reprogramming. ${ }^{133}$ Reprogrammers use the model to visualize shared background assumptions, express generalizations about experiments, and correlate cell state and developmental potential. These uses highlight several contrasts with Waddington's original model. First, stem cell biologists interpret the developing entity as a cell. Given this assumption, which Waddington allows for, but does not make himself, the landscape model visualizes key features of the stem cell concept: a single undifferentiated starting point, with the potential to develop along a variety of pathways, gradually restricted as development proceeds, and ending with stable, mature cell types.

A second, related contrast concerns 'units' of stability and determination. For Waddington, these are developmental pathways: "it is the track 
as a whole which, compared with any line lying between the tracks, is a description of an equilibrium" $(1940,92)$. He explicitly denies that points on the landscape represent equilibrium states of tissues. Instead, networks of interacting genes and gene products determine robust pathways, and pieces of tissue develop via a sequence of robust tracks punctuated by binary 'decisions.' Stem cell biologists, in contrast, take points on the landscape to represent cell states, which are more or less stable with respect to intervention. On this interpretation, the rolling ball represents a cell passing through different states in a process of differentiation. Under these assumptions, Waddington's argument that genes collectively determine the form of developmental pathways no longer applies. Moreover, the representation of gene action does not structurally correspond to that of development. These altered representational assumptions omit Waddington's rationale for the hypothesis that genes are the underlying determinants of development.

Another contrast concerns the relation of development and evolution. Stem cell biologists are, for the most part, unconcerned with evolutionary processes. Instead, they focus on specific mechanisms operating during an organism's lifetime or in the transparent, simplified 'bodies' of cell culture. They do not attempt to explain long-term changes in organismal populations, nor the gradual sculpting of adaptations, nor interspecies relations. So stem cell biologists do not share Waddington's rationale for treating the landscape as malleable. However, the landscape could be conceived dynamically on developmental timescales. Changes in topography could be induced by cell movement, experimental manipulation, or random 'noise' in cellular systems. Importantly, DNA sequences can today be altered as easily as other components of developmental mechanisms. So there is no reason to represent genes as uniquely stable or fixed. Instead, stem cell biologists treat the entire landscape as a fixed background for representing changes in cell state and potential (Figures 6.5 and 6.6).

In recent diagrams, the landscape provides a backdrop for generalizations about reprogramming experiments and hypotheses about their relation to normal development. Developmental processes are depicted as arrows describing trajectories on the landscape. In Figure 6.5, for example, normal development is visualized as a trajectory down the landscape, reprogramming as the reverse. More elaborate summaries compare and contrast different reprogramming experiments. In Figure 6.6, Pathway 1 shows "complete" reprogramming: conversion of a differentiated cell to a stable pluripotent state. Pathway 2 depicts "incomplete" reprogramming, in which a differentiated cell is temporarily converted to a pluripotent 
(a) Development

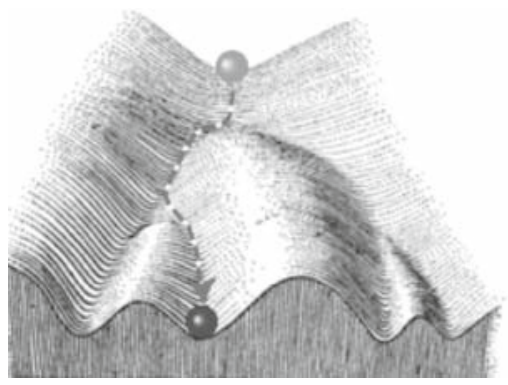

(b) Pluripotent reprogramming (SCNT, iPS)

Figure 6.5 Waddington's landscape co-opted to represent reprogramming experiments. (a) Normal cell development as a trajectory down the landscape. (b) Reprogramming visualized as a reversal of normal development. Reprinted from Zhou and Melton (2008) with permission of Elsevier Press

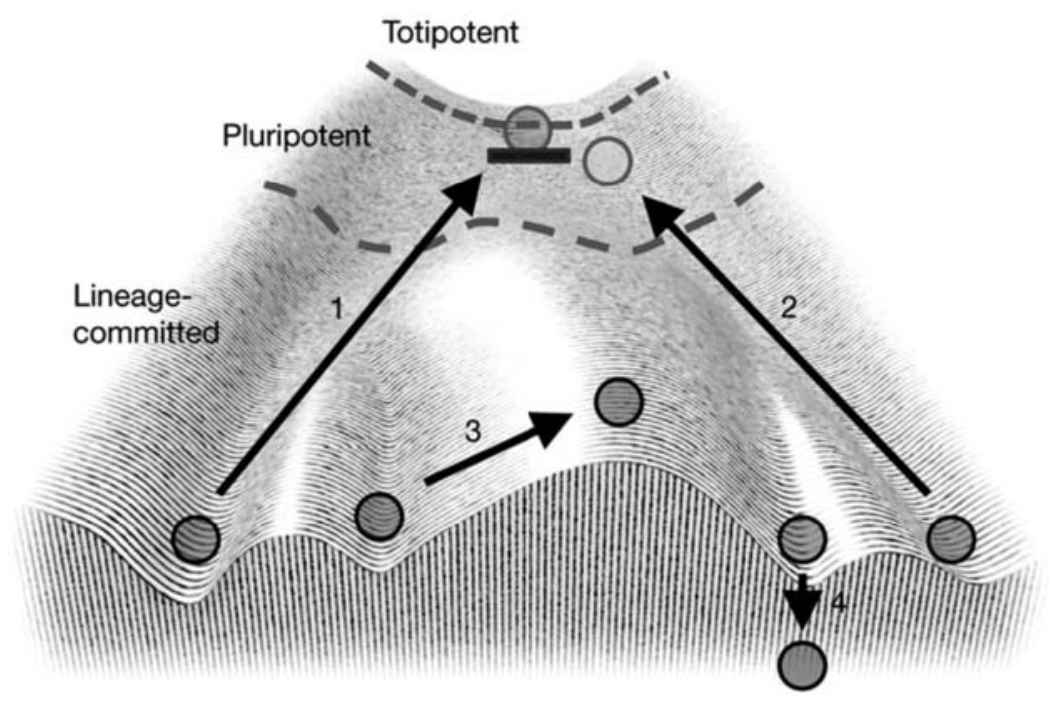

Figure 6.6 Waddington's landscape as the background for experimental manipulations of cell fate and potential. Reprinted from Yamanaka (2009) by permission of Macmillan Publishers Ltd: Nature 
state, but normal development then resumes. Pathway 3 represents "transdifferentiation:" direct conversion from one differentiated cell type to another without passing through a pluripotent state. Finally, Pathway 4 depicts cell death $-\mathrm{a}$ frequent outcome of experimental manipulations, and also a 'programmed' response of cells in a variety of circumstances. Each pathway is a departure from normal development.

These modified landscape diagrams represent generalizations about reprogramming experiments. Just as Waddington generalized from experimental manipulations of embryonic development in fly, frog, and chick, abstracting details to highlight formal features of developmental processes, cell reprogrammers generalize from experimental manipulations of cell development. But while Waddington inferred formal features of organismal development from the results of experiments, stem cell biologists infer formal features of experimental methods themselves as manipulations of normal development. Different classes of reprogramming experiment are distinguished not by methodological details, but by the tracks they describe on the landscape; each a different deviation from normal development. In this way, the landscape model compresses thousands of reprogramming experiments into a few generalizations. Visualizing these on the landscape suggests possible explanations for experimental outcomes.

One generalization that has emerged from six years of reprogramming is that the success rate within an experiment (i.e. percentage of treated cells that become pluripotent) is inversely correlated with the source organism's age. For example, producing iPSC from cells of an adult is far less efficient than producing ESC from cells of an embryo. The modified landscape diagrams sketch an explanation: reprogramming "pushes" cells up the developmental incline, reversing the paths traced by their prior development (Figure 6.5). The source of the push is unspecified: it could be the experimenter, specific proteins, specific genes, or some combination thereof. The hypothesis represented is that the more differentiated the cell, the further "uphill" it must travel to reach a pluripotent state and the more likely it is that some other factor will block its path. Another experimental generalization is that pluripotency is unstable in most cells; most reprogramming is "incomplete." Reprogrammers speculate that complete, stable reprogramming requires an "epigenetic bump" to prevent the cell from rolling back down the hill once the inducing stimulus, whatever its nature, is removed (Yamanaka 2009, 50). The need for the extra "block" is shown as a dark bar on the landscape - a tiny 'black box' (Figure 6.6).

More detailed hypotheses elaborate on these sketches. But, strikingly, none appeal to the underside of Waddington's landscape, with genes 
as the "ultimate determinants" of developmental topography. Indeed, DNA sequences are seldom discussed. Reprogrammers look instead to other components of molecular mechanisms to explain generalizations represented on the landscape. One idea is that chemical modifications of DNA and chromosomal proteins progressively accumulate during development (see, for example, Zhou and Melton 2008, 386). The greater the portion of the epigenome needing to be "wiped clean," the greater the difficulty and the less likely the process is to succeed. Another proposal is that regulatory binding sites on nuclear DNA randomly shift from "open" to "closed" positions and vice versa. The few cells induced to pluripotency are just those with the right regulatory binding sites "open" at the right time (Hochedlinger and Plath 2008, Yamanaka 2009).

Intuitions underlying these proto-explanations are complicated, as background assumptions about development are entangled with twentieth-century gene-centrism. The basic principles of development, as visualized in the landscape, include unidirectionality and progressive restriction of developmental potential. These principles preserve the traditional idea that development is irreversible, presupposed in much embryological thought, and carried forward into developmental biology. The notion that genes are fundamental for biological explanation encourages the view that genetic changes to nuclear DNA are irreversible (Keller 2002). All other kinds of changes, lumped together as "epigenetic," must then be reversible, at least in principle. The process of organismal development (for the most part) consists of epigenetic changes, as early reprogramming experiments demonstrate. Reprogramming is thus the artificial exception that proves the normal rule, that development is irreversible, by exploiting its in principle reversibility. Entrenched association of epigenetics with reversibility of development makes it intuitively plausible to suppose that reprogramming is just development in reverse, as depicted on the landscape. But this assumption rests on ideas about genetic primacy that are not generally accepted in stem cell biology. So particular care is needed when interpreting reprogramming experiments, to avoid bias in favor of this unmotivated supposition. With this caveat in mind, Waddington's landscape sets the stage for MEx of developmental phenomena more generally.

This is accomplished in the model's third use by stem cell biologists: correlating cell state and developmental potential. As discussed in previous chapters, stem cell experiments measure both cells' molecular traits and their developmental potential. An array of such experiments correlates the two sets of measurements. Waddington's landscape is a helpful device for visualizing this correlation (Figure 6.7). The higher 


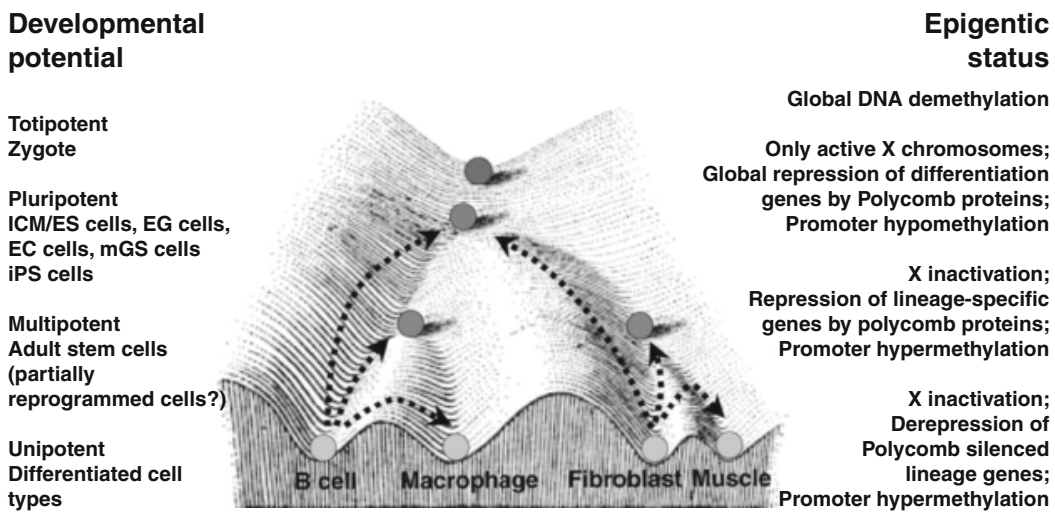

Figure 6.7 Correlation of cell state and developmental potential via the landscape model. Reprinted from Hochedlinger and Plath (2009) with permission from Development

a cell's position on the slope, the greater its developmental potential. This position, representing cell state, can then be associated with a pattern of gene expression, constituted by interacting DNA, RNA, protein, and small molecules. Experimental manipulation of the molecular components of regulatory networks reveals the details of these mechanisms. The landscape model thus offers a convenient coordinating framework, to be filled out by further experiments.

\subsection{Regulatory genes}

So far, I have argued that, in experimental biology in general and stem cell biology in particular, genes are better understood as vital components of complex molecular mechanisms than "master molecules" controlling development. In this final section I consider an influential opposing view: "the regulatory genome" (Davidson 2006, Davidson and Levine 2008). On this view, development is controlled by a DNA-encoded program made up of short sequences that specifically bind TF proteins and thereby make a difference to gene expression. Davidson terms these DNA sequences "cis-regulatory modules." Each module's effect is represented as a conditional rule of the form: "If protein $\mathrm{X}$ is present, then gene $\mathrm{Y}$ is expressed at level Z. ${ }^{134}$ Each gene has a set of such modules associated with it, which collectively specify its expression pattern under various conditions. So control of development is attributed entirely to the DNA components of molecular complexes that make a 
difference to gene expression. Protein components of these complexes are conceived as "inputs" to the information-processing modules and effects on gene expression as "outputs."

These basic "cis-regulatory" units are organized into systems of interacting modules. The inputs that determine gene expression at any given time, mainly TF protein concentrations, comprise a "regulatory state." For every gene in every nucleated cell, regulatory DNA modules process information about the cell's regulatory state into effects on gene expression. Of course, TF proteins are themselves products of gene expression. The regulatory modules that control expression of TF genes are sites of "primary core control" for development. Because TFs influence one another's expression, both positively and negatively, the "core control" modules form an interconnected network. So, for any organism, the genetic regulatory network (GRN), composed of DNA sequences distributed throughout its genome, constitutes a stable underlying program for development.

DNA, though not genes in the traditional protein-encoding sense, therefore explains development:

The design features of the GRN directly explain why the events of a given process of development occur; for example, why a given set of cells becomes specified to a specific fate, why it emits particular signals to adjacent cells, and why it differentiates in a given direction. The architecture of a GRN is mandated by the cis-regulatory sequences...that control each gene of the network. These sequences determine what inputs affect expression of each gene, and how these inputs operate in a combinatorial fashion.

(Davidson and Levine 2008, 20063)

Collectively, DNA modules act as "a vast, delocalized computational device" that processes regulatory states of a cell (Davidson 2006, 185). These sequences are (for the most part) invariant across cells of an organism and organisms of a species, and a relatively small set of TF is conserved throughout much of the animal kingdom. GRNs, therefore, offer a unified explanation of animal evolution and development.

The regulatory genome is an explanatory model based on experimental results accumulated over several decades, which revealed core mechanisms of early development in a few model organisms (notably sea urchin and Drosophila). Essentially, Davidson and colleagues interpreted this enormously detailed and complex dataset in light of the assumption that DNA controls development. By omitting or drastically simplifying other 
components, they produced a more tractable model, which they then extrapolated to animal development in general. There is nothing problematic about this modeling approach. The question here is whether the basic assumption, that DNA controls development, is justified in stem cell biology. Several points made above support a negative answer. First, gene expression, which is central to explanations of stem cell phenomena, involves many components besides DNA. Because DNA sequences are (for the most part) invariant across cells of an organism, they cannot be actual difference-makers for gene expression. So there is no reason to privilege DNA as a cause of development. GRN models actually reflect this: features of non-DNA components that make a difference to gene expression are represented in compressed form, as arrows linking regulatory DNA sequences. The causally active components of GRNs are not limited to DNA sequences.

Nor can DNA claim causal priority in virtue of being the initial step in the linear chain, DNA $\rightarrow$ RNA $\rightarrow$ protein. As a causal model, the Central Dogma has been superseded by interactive networks in which diverse TF are implicated in the expression of any particular gene, TF proteins have context-dependent effects, and micro-RNAs influence gene expression and protein activity. Within such a network, no single position can be non-arbitrarily identified as the start-point. It follows that DNA cannot occupy such a position. Even at the earliest stages of an organism's existence, DNA, RNA, proteins, and small molecules are organized in elaborate, ongoing interactions. Nor is DNA set apart from other network components by its informational properties. GRNs do not involve coding relations like those linking terms of the Central Dogma, namely that DNA sequence is a template for RNA sequence, which is, in turn, a template for an amino acid sequence. The linguistic terms 'transcription' and 'translation' refer to the molecule-by-molecule mapping that is conceptualized as a code. In contrast, regulatory modules in genomic DNA specifically bind proteins not as templates that allow mapping between sequences, but via molecular bonds mediated by conformational and electrochemical properties - just as complexes of proteins and RNA do.

So the role of DNA sequences in gene expression is not distinctive, either causally or informationally. However, DNA is distinguished from other components of developmental regulatory mechanisms by invariance within an organism. DNA sequences can therefore provide a stable, underlying architecture for development. For Davidson, committed to a unified explanation of evolution and development, this is a crucial point. Regulatory DNA sequences differ across species and so are actual 
difference-makers for development in comparative perspective. These explanatory aims motivate the assumption of genetic control. But stem cell biology is concerned with the lives of individual organisms within species. The two explanatory projects are distinct. Davidson's account is intended to provide a unified explanation of development and evolution, with particular emphasis on variation among species, and focuses on development of the whole organism. Stem cell biologists, in contrast, aim to explain development at the cellular level, with particular emphasis on variation within an organism. The regulatory gene model therefore plays a minimal role in explanations of stem cell phenomena, while stem cell phenomena are explicitly omitted from Davidson's account. 135

The aims of stem cell biology do not rule out attributing explanatory importance to invariant DNA sequences within an organism. Their explanatory role cannot, however, be one of causal control. Instead, stem cell biologists might privilege regulatory genes as stable coordinators of diverse molecular interactions. The controlling gene would then give way to the coordinating gene in explanations of stem cell phenomena.

\subsection{Conclusion}

This chapter criticizes the view that genes have a privileged role in explanations of development. The causal-informational gene concept has been criticized before, in light of the paradox of development and the rising tide of molecular complexity. However, focus on stem cells brings new dimensions to the debate. Philosophical accounts of genes in development tend to frame the issue in terms of molecules and organisms, neglecting the mediating levels of cells, tissues, and organs. The latter, however, comprise the major domains of stem cell phenomena. In particular, iPSC and cell reprogramming offer a valuable test case for Waters' account of genes as privileged difference-makers in development. Close examination shows that DNA sequences are not actual specific difference-makers in stem cell experiments. Instead, reprogramming experiments aim to reveal interacting, joint causes of developmental pathways. These results further support the joint account of MEx, presented in Chapter 5, which is incompatible with genetic explanatory privilege.

These negative arguments motivate an alternative account of the role of genes in development, which is provided by Waddington's landscape. The latter model is particularly apropos, as it was originally intended to integrate ideas about genetics and development and has recently been co-opted by stem cell biologists to represent experimental results and 
speculative explanations thereof. Waddington's original model had three purposes: to represent developmental pathways, express the hypothesis that genes indirectly control development through a network of interacting biochemical products, and 'visually unify' genetics, development, and evolution. Stem cell biologists update the landscape model to serve somewhat different purposes, one consequence of which is "parity" of genes with other components of gene expression mechanisms. Waddington's model, with these modifications, offers a framework for MEx of cell development. Finally, an influential alternative, which identifies a subset of DNA sequences as the "core program" controlling development, is shown to be peripheral to stem cell biology today. 